\title{
Paro cardiorrespiratorio intraoperatorio en un paciente con síndrome de Brugada
}

\author{
Intraoperative cardiac arrest in a patient with \\ Brugada syndrome
}

Ignacia López L. ${ }^{1}$, Claudio Pacheco C. ${ }^{1}$, Cristóbal López L. ${ }^{2}$, Francisco Cruzat R. ${ }^{3}$

\begin{abstract}
A 61-year-old female patient with history of hipertension is scheduled to undergo a minor ginecological procedure (endoscopic endometrial polipus resection) with general anesthesia. She received standard monitorization, induction with midazolam, propofol and fentanyl. Ventilated with laringeal mask. Anesthesia was maintained with sevoflurane, nitrous oxide and oxygen. During surgical procedure, the patient received atropine and ephedrine associated with two episodes of bradycardia without hemodinamic disturbances. The surgery ended without problems. During the weaking up process she presented characteristical waves of ventricular fibrillation, recuperating sinusal rhythm secondary to defibrillation with $360 \mathrm{~J}$. There was no clear cause for cardiac arrest at that moment so patient was translated to the ICU for observation, monitoring and study. Postoperative EKG presented an ascending ST segment in $V_{1}$ to $V_{3}$ derivations without hemodynamic alterations associated. The possible diagnosis of Brugada's Syndrome was proposed. The patient received an implantable defibrillator. The mechanisms and anesthetic implications are discussed and reviewed.
\end{abstract}

\section{RESUMEN}

Paciente de 61 años, hipertensa, fue sometida a un procedimiento endoscópico menor (histeroscopía) bajo anestesia general balanceada. Recibió monitorización estándar, inducción con midazolam, propofol y fentanilo. Se ventiló con máscara laríngea y la mantención anestésica fue con sevoflurano asociado
Key words:

Brugada syndrome, anesthesiology, cardiac arrest

\section{Palabras clave:}

Paro cardiorrespiratorio, síndrome de Brugada, anestesiología

Interno, Facultad de Medicina, Universidad de los Andes.

Alumno Medicina, Facultad de Medicina, Pontificia Universidad Católica de Chile.

División de Anestesiología, Pontificia Universidad Católica de Chile.

Fecha de recepción: 27 de agosto de 2019

Fecha de aceptación: 17 de septiembre de 2019

\section{ORCID}

https://orcid.org/0000-0001-7196-5174

Correspondencia:

Francisco Cruzat R.

Email: fjcruzatr@gmail.com 
a $\mathrm{N}_{2} \mathrm{O}$ en $\mathrm{O}_{2}$. En dos oportunidades recibió atropina y efedrina para el manejo de bradicardias sin compromiso hemodinámico. Se completó el procedimiento ginecológico sin complicaciones. Durante el despertar anestésico, presentó una fibrilación ventricular que cedió con desfibrilación. Se estabilizó y trasladó a UCI donde se estudió las posibles etiologías. Se obtiene ECG postoperatorio con elevación del segmento ST en derivaciones $V_{1}$ a $V_{3}$ sin alteraciones hemodinámicas asociadas, postulándose un síndrome de Brugada. Se le instaló desfibrilador implantable. Se revisa y discuten los diversos mecanismos e implicancias anestésicas asociadas.

\section{Introducción}

A diferencia de lo que ocurre en el ambiente extrahospitalario, el paro cardiorrespiratorio (PCR) en pabellón, permite un diagnóstico y tratamiento precoz. Para esto, es necesario tener un adecuado control de la situación, acceso inmediato a drogas y a un desfibrilador. En este reporte, describimos el caso de una paciente sometida a procedimiento ginecológico menor bajo anestesia general, que durante el despertar anestésico presentó un PCR sin causa aparente. La desfibrilación precoz permitió la recuperación del ritmo y un buen desenlace postoperatorio.

\section{Caso clínico}

Paciente de 61 años de edad, ASA II (por hipertensión arterial en tratamiento) sometida a una resección electiva de pólipos endometriales por vía endoscópica.

Luego de la monitorización estándar (presión arterial no invasiva, oximetría de pulso y electrocardiografía continua), se indujo una anestesia general con midazolam $5 \mathrm{mg}$, propofol $150 \mathrm{mg}$ y $150 \mu \mathrm{g}$ de fentanilo. Se instaló una máscara laríngea y se conectó a ventilación mecánica (VM). La mantención anestésica fue con sevoflurano con gas espirado menor a 1 $\mathrm{MAC}$, en mezcla con $\mathrm{N}_{2} \mathrm{O}$. A los 20 minutos de iniciada la cirugía, recibió $0,1 \mathrm{mg}$ de atropina y $16 \mathrm{mg}$ de efedrina por bradicardia hasta 40 latidos por minuto sin disminución de la presión arterial media. A los 30 minutos, se inyectó nuevamente 0,2 mg de atropina por un nuevo episodio de bradicardia, con lo cual la frecuencia cardíaca volvió a los valores basales $(\approx 55$ latidos por minuto). La paciente recibió dexametasona $8 \mathrm{mg}$ como profilaxis de náuseas y vómitos[16], ketorolaco $60 \mathrm{mg}$ y $1.500 \mathrm{ml}$ de suero Ringer Lactato como hidratación en el intraoperatorio.
La cirugía finalizó exitosamente y con balance hídrico neutro. Se procedió a despertar a la paciente al cabo de 55 minutos desde el inicio de la cirugía. Mientras se esperaba el despertar espontáneo de la paciente, sin mediar estímulo alguno, el monitor (derivación DII) mostró ondas características de fibrilación ventricular. Se tomó el pulso a la paciente, sin detectarlo, por lo que se iniciaron maniobras de reanimación con masaje cardiaco externo continuo $(\approx 100$ por minuto). Segundos después se procedió a dar una descarga de 360 Joules con el desfibrilador monofásico, logrando ritmo sinusal y pulso. Se administró 1 $\mathrm{mg}$ de epinefrina y $80 \mathrm{mg}$ de lidocaína. Se aseguró la vía aérea con tuboendotraqueal (TOT) y se conectó a VM. Se tomaron muestras de sangre para análisis. Se realizó ecocardiografía transesofágica intraoperatoria encontrándose hipoquinesia septoapical.

Hasta ese momento no había causa evidente del $P C R$, por lo que, una vez estabilizada la paciente, se decide traslado a Unidad de Cuidados Intensivos (UCI) para mantener monitorización, observación y estudio de la etiología del cuadro.

Una vez en $\mathrm{UCl}$, se administró carga con amiodarona y se plantearon diversos diagnósticos diferenciales; síndrome coronario agudo, miocardiopatía hipertrófica, valvulopatía, entre otras. Se realizaron electrocardiogramas (ECG) seriados y se solicitó evaluación por equipo de cardiología. El ECG basal perioperatorio mostraba bloqueo incompleto de rama derecha con signos de sobrecarga ventricular izquierda y luego del evento el ECG señalaba leve ascenso de segmento $S T$ en derivaciones $V_{1} V_{2} V_{3}$ (menor a 1,5 $\mathrm{mm}$ ) sin alteraciones hemodinámicas asociadas. Los exámenes sanguíneos no mostraban alteraciones hidroelectrolíticas mayores y la troponina I fue de $2 \mathrm{pg} /$ $\mathrm{mL}$ (valor normal $<14 \mathrm{pg} / \mathrm{mL}$ ). Se propone el diagnóstico de síndrome de Brugada.

A las 3 horas del evento, la paciente se encontraba en condiciones de extubación óptimas por lo que se decide el retiro del TOT, y el traslado a la Unidad 
Coronaria. Fue manejada con propranolol $(20 \mathrm{mg} / 6$ h), AAS (100 mg/día), losartán ( $25 \mathrm{mg} / 12 \mathrm{~h})$, amiodarona $(200 \mathrm{mg} / 8 \mathrm{~h}$ ) y se programa para coronariografía.

En Unidad Coronaria se mantiene en observación por $72 \mathrm{~h}$ manteniéndose asintomática y se realiza una ecocardiografía control que concluye: ventrículo izquierdo no dilatado, paredes de grosor normal, sin alteraciones claras de motilidad segmentaria. Fracción de eyección de 60\%. Disfunción diastólica tipo 1 con dilatación leve de aurícula izquierda.

Posteriormente, se realizó coronariografía que mostró ausencia de lesiones y se indica desfibrilador VVI que se instala en otro centro al cual es trasladada.

\section{Discusión}

El síndrome de Brugada es una canalopatía, con una prevalencia de 0,1 a $1 \%$, caracterizada por un patrón donde se evidencia elevación del segmento ST ( $\geq 2 \mathrm{~mm}$ ) asociado o no a bloqueo de rama derecha, seguido de una onda T negativa $\geq 1 \mathrm{~mm}$ en las derivaciones precordiales derechas $V_{1}$ a $V_{2}[1]$.

Típicamente se manifiesta en la adultez, alrededor de la cuarta década de la vida, afectando mayoritariamente al sexo masculino, principalmente caucásicos y asiáticos. Alrededor del $20 \%$ de los pacientes tiene antecedentes familiares de síncope, FV documentada o de muerte súbita de probable origen cardíaco[2].

Sería secundario a defectos genéticos de la subunidad $\alpha$ de los canales de sodio presentes en el corazón. La mutación más conocida es la que afecta al gen SCN5A ubicado en el cromosoma 3, la cual se transmite de forma autosómica dominante. Esta mutación lleva a una disminución de la corriente rápida de sodio durante el potencial de acción[3],[4].

Las manifestaciones al ECG del síndrome de Brugada pueden ser moduladas por los bloqueadores de canales de sodio, estados febriles o de hipotermia, agentes vagotónicos, agonistas $\alpha$ adrenérgicos, $\beta$ bloqueadores, antidepresivos tricíclicos o tetracíclicos, la hiperkalemia, hipercalcemia, la combinación de glucosa e insulina y el uso de alcohol y de cocaína[5].

Los pacientes con síndrome de Brugada que han presentado síncopes o episodios de muerte súbita recuperada tienen un peor pronóstico sin tratamiento. Aquellos pacientes cuyo diagnóstico ha sido incidental, por un ECG compatible con el síndrome, que están asintomáticos y no tienen antecedentes familiares de muerte súbita suelen tener un curso más benigno. Esto ha permitido estratificar el riesgo de muerte y es una información que debiese obtenerse en la evalua- ción preanestésica[6].

El único tratamiento que ha demostrado mejorar la sobrevida ha sido el desfibrilador implantable. Los antiarrítmicos han sido de poco ayuda en la prevención de la TV y la FV. La mortalidad anual de estos pacientes es de $10 \%$, sean estos sintomáticos o asintomáticos[7].

Las consideraciones anestésicas para los pacientes con síndrome de Brugada aún no han sido establecidas por los escasos reportes al respecto. Lo ideal sería contar con una evaluación cardiológica si se tiene algún antecedente familiar que sugiera esta patología.

En estos pacientes está contraindicado el uso de drogas que bloqueen los canales de sodio, como la procainamida y flecainamida. Algunos agonistas $\alpha$ adrenérgicos pueden aumentar el supradesnivel del ST, así como también la neostigmina y los antiarrítmicos de tipo IA, por esta razón no es prudente antagonizar el bloqueo neuromuscular[8],[9],[10] a pesar de que existen publicaciones donde se ha realizado sin inconvenientes[11],[12].

Los pacientes con síndrome de Brugada también están en riesgo de desarrollar otras arritmias distintas a la TV y la FV, lo cual debe ser considerado en su manejo anestésico[13]. Este potencial arritmogénico hace parecer adecuado el contar con un desfibrilador en la sala de operaciones y más aún el tener los parches autoadhesivos para desfibrilación puestas sobre el paciente si es que existe el antecedente de FV.

La monitorización de los pacientes con síndrome de Brugada debiese incluir, además de la monitorización básica, evaluación del segmento ST en el ECG de al menos 3 derivadas, una de ellas derecha y medición invasiva de presión arterial y temperatura[14].

El uso de anestésicos inhalatorios no ha demostrado desencadenar arritmias en las escasas publicaciones al respecto[9],[11],[12].

Los cambios en el tono autonómico, principalmente aquellos que resultan en bradicardia, desencadenan con facilidad arritmias malignas, por lo tanto, se hace necesario el uso de atropina cuando la frecuencia cardiaca cae a menos de 60 por minuto y una analgesia postoperatoria adecuada, con una combinación de opioides endovenosos y antiinflamatorios no esteroidales o evitando utilizar grandes dosis de anestésicos locales en caso de anestesia regional[15].

La anestesia regional induciría con menor frecuencia cambios autonómicos en comparación con la anestesia general y puede ofrecer una buena analgesia postoperatoria, pero debemos considerar que los bloqueadores de canales de sodio pueden inducir taquiarritmias[16]; por lo tanto, su administración debe ser cuidadosa. 
Considerando que las arritmias ocurren con mayor frecuencia en el periodo postoperatorio, se hace necesario realizar la recuperación en una unidad de cuidados intensivos para su detección precoz. Algunos estudios recomiendan este monitoreo por hasta 36 horas[14].

En conclusión, se hace necesario un alto índice de sospecha en aquellos pacientes que cuenten con antecedentes que nos sugieran estar frente a esta patología, tomar las precauciones necesarias para evitar o manejar adecuadamente las arritmias que pudieran desencadenarse en la sala de operaciones y una gran cooperación entre anestesistas y cardiólogos para el adecuado manejo pre y postoperatorio de estos pacientes.

\section{Referencias}

1. Bayés de Luna $A$, Brugada J, Baranchuk A, Borggrefe M, Breithardt G, Goldwasser D, et al. Current electrocardiographic criteria for diagnosis of Brugada pattern: a consensus report. J Electrocardiol. 2012 Sep;45(5):433-42. https://doi.org/10.1016/j. jelectrocard.2012.06.004 PMID:22920782

2. Priori $S G$, Wilde $A A$, Horie $M$, Cho Y, Behr ER, Berul C, et al. HRS/EHRA/APHRS expert consensus statement on the diagnosis and management of patients with inherited primary arrhythmia syndromes: document endorsed by HRS, EHRA, and APHRS in May 2013 and by ACCF, AHA, PACES, and AEPC in June 2013. Heart Rhythm. 2013 Dec;10(12):193263. https://doi.org/10.1016/j. hrthm.2013.05.014 PMID:24011539

3. Priori SG, Napolitano C, Gasparini $\mathrm{M}$, Pappone C, Della Bella P, Giordano U, et al. Natural history of Brugada syndrome: insights for risk stratification and management. Circulation. 2002 Mar;105(11):1342-7. https://doi. org/10.1161/hc1102.105288 PMID:11901046

4. Hedley PL, Jørgensen P, Schlamowitz S, Wangari R, MoolmanSmook J, Brink PA, et al. The genetic basis of long QT and short QT syndromes: a muta- tion update. Hum Mutat. 2009 Nov;30(11):1486-511. https:// doi.org/10.1002/humu.21106 PMID:19862833

5. Antzelevitch C, Brugada P, Borggrefe $M$, Brugada J, Brugada R, Corrado D, et al. Brugada syndrome: report of the second consensus conference: endorsed by the Heart Rhythm Society and the European Heart Rhythm Association. Circulation. 2005 Feb;111(5):659-70. https://doi.org/10.1161/01. CIR.0000152479.54298.51 PMID:15655131

6. Sahinkaya HH, Yasar E, Tekgül ZT, Horsanalı BÖ, Özeroglu E. Anaesthetic Management of a Patient with Brugada Syndrome. Turk J Anaesthesiol Reanim. 2016 Apr;44(2):96-8. https://doi. org/10.5152/TJAR.2016.22230 PMID:27366566

7. Al-Khatib SM, Stevenson WG, Ackerman MJ, Bryant WJ, Callans DJ, Curtis AB, et al. 2017 AHA/ACC/HRS guideline for management of patients with ventricular arrhythmias and the prevention of sudden cardiac death: Executive summary: A Report of the American College of Cardiology/American Heart Association Task Force on Clinical Practice Guidelines and the Heart Rhythm Society. Heart Rhythm. 2018 Oct;15(10):e190-252. https://doi.org/10.1016/j. hrthm.2017.10.035 PMID:29097320

8. Kim JS, Park SY, Min SK, Kim
JH, Lee SY, Moon BK, et al. Anaesthesia in patients with Brugada syndrome. Acta Anaesthesiologica Scandinavica. Wiley; 2004 Sep;48(8):1058-61. http://dx.doi.org/10.1111/j.00015172.2004.00470.x

9. Inamura $\mathrm{M}$, Okamoto $\mathrm{H}, \mathrm{Ku}$ roiwa M, Hoka S. General anesthesia for patients with Brugada syndrome. A report of six cases. Can J Anaesth. 2005 Apr;52(4):409-12. https:// doi.org/10.1007/BF03016285 PMID:15814757

10. Postema PG. Wolpert C, Amin AS, et al. Drugs and Brugada syndrome patients: review of the literature, recommendations, and an up-to-date website (www.brugadadrugs.org). Heart Rhythm 2009; 6: 1335-41.

11. Candiotti KA, Mehta V. Perioperative approach to a patient with Brugada syndrome. J Clin Anesth. 2004 Nov; 16(7):529-32. https://doi.org/10.1016/j.jclinane.2003.09.018 PMID:15590257

12. Edge CJ, Blackman DJ, Gupta K, Sainsbury M; Edge CJ. Blackman DJ, Gupta K, Sainsbury M. General anaesthesia in a patient with Brugada syndrome. Br J Anaesth. 2002;89(5):788-91. https://doi. org/10.1093/bja/89.5.788.

13. Morita H, Zipes DP, Wu J. Brugada syndrome: insights of ST elevation, arrhythmogenicity, and risk stratification from experimental observations. Heart Rhythm. 2009 Nov;6(11 Suppl):S34-43. 
https://doi.org/10.1016/j.

hrthm.2009.07.018

PMID:19880072

14. Santambrogio LG, Mencherini S, Fuardo M, Caramella F, Braschi A. The surgical patient with Brugada syndrome: a fourcase clinical experience. Anesth Analg. 2005 May;100(5):12636. https://doi.org/10.1213/01. ANE.0000149327.23267.6B

\section{PMID:15845665}

15. Gan TJ, Meyer T, Apfel CC, Chung F, Davis PJ, Eubanks S, et al.; Department of Anesthesiology, Duke University Medical Center. Consensus guidelines for managing postoperative nausea and vomiting. Anesth Analg. 2003 Jul;97(1):62-71. https://doi.org/10.1213/01.
ANE.0000068580.00245.95

PMID:12818945

16. Kloesel B, Ackerman M, Sprung $J$, et al. Anesthetic management of patients with Brugada syndrome: a case series and literature review. Canadian Journal of Anesthesia/Journal canadien d'anesthésie. 2011;58(9):824836. https://doi.org/10.1007/ s12630-011-9546-y 